\title{
Ultralow Loss, High $Q$, Four Port Resonant Couplers for Quantum Optics and Photonics
}

\author{
H. Rokhsari and K. J. Vahala \\ Thomas J. Watson Laboratory of Applied Physics, California Institute of Technology, Pasadena, California 91125, USA
}

(Received 2 December 2003; published 25 June 2004)

\begin{abstract}
We demonstrate a low-loss, optical four port resonant coupler (add-drop geometry), using ultrahigh $Q\left(>10^{8}\right)$ toroidal microcavities. Different regimes of operation are investigated by variation of coupling between resonator and fiber taper waveguides. As a result, waveguide-to-waveguide power transfer efficiency of $93 \%(0.3 \mathrm{~dB}$ loss $)$ and nonresonant insertion loss of $0.02 \%(<0.001 \mathrm{~dB})$ for narrow bandwidth $(57 \mathrm{MHz})$ four port couplers are achieved in this work. The combination of low-loss, fiber compatibility, and wafer-scale design would be suitable for a variety of applications ranging from quantum optics to photonic networks.
\end{abstract}

DOI: 10.1103/PhysRevLett.92.253905

Minimizing optical loss is of crucial importance in various studies, as it is often the main obstacle in realizing distinct physical functionalities. This is true in quantum optical applications of microcavities where parasitic loss can both inhibit the generation of quantum states and interfere with intended coupling to a transport medium such as optical fiber [1-8]. Also, in photonic applications of these devices [9-12] the ability to attain high efficiency power transfer between two distinct waveguides is of great interest. The ability to attain coupling coefficients between the resonator and waveguides that are greater than the intrinsic round-trip loss of the cavity (usually called the overcoupled regime) is fundamental to achieve high waveguide-to-waveguide resonant power transfer efficiency. High waveguide coupling efficiency and high intrinsic quality factor are hence essential in all applications of waveguide-coupled resonator systems. Such characteristics ensure that the overall quality factor of the system can be dominated by the intentional control of waveguide loading (coupling into and out from the resonator) as opposed to parasitic mechanisms which include intrinsic losses of the cavity and scattering losses at the waveguide-resonator junctions. Ultrahigh-qualityfactor (UHQ) microresonators $\left(Q>10^{8}\right)$ have been extensively studied $[7,8,13]$, and the ability to provide high efficiency coupling to UHQ devices by use of low-loss tapered fiber waveguides has also been verified [14]. In this Letter, we explore a new realm of performance enabled by the combination of these results. Toroidal microcavities with intrinsic $Q$ factors in excess of $10^{8}$ were fabricated and studied in the so-called add-drop geometry where a whispering-gallery mode enables resonant power transfer between two distinct waveguides. Greater than $93 \%$ power transfer efficiency is measured in devices having loaded $Q$ factors of $3.3 \times 10^{6}$ (overall quality factor of the resonator including coupling to waveguides), while the nonresonant insertion loss remains less than $0.02 \%$. The transfer efficiency is predicted to be even higher for lower loaded $Q$ values. This represents a substantial improvement compared to all prior
PACS numbers: 42.79.Gn, 42.60.Da, 42.82.Et, 42.79.Sz

work on similar microcavity-type structures, elevating their performance to a level at which they could be useful for loss-sensitive quantum optical applications which until now have not been accessible for four port resonant couplers. A model of the resonant-coupling efficiency and quality factor variation versus waveguide loading is also confirmed by adjusting the coupling between the resonator and waveguides.

Figure 1 depicts the device geometry including a UHQ microtoroid resonator evanescently side coupled to a pair of tapered optical fibers. This configuration allows the microcavity to act as a frequency selective coupler. Resonant optical power ( $\lambda_{2}$ in the figure) launched into port 1 is transferred to port 4 via the microtoroid, while nonresonant waves are largely unaffected upon transmission beyond the resonator-waveguide junction. This system (symmetric or asymmetric geometry add-drop [15]) can be studied using a simple model based on the

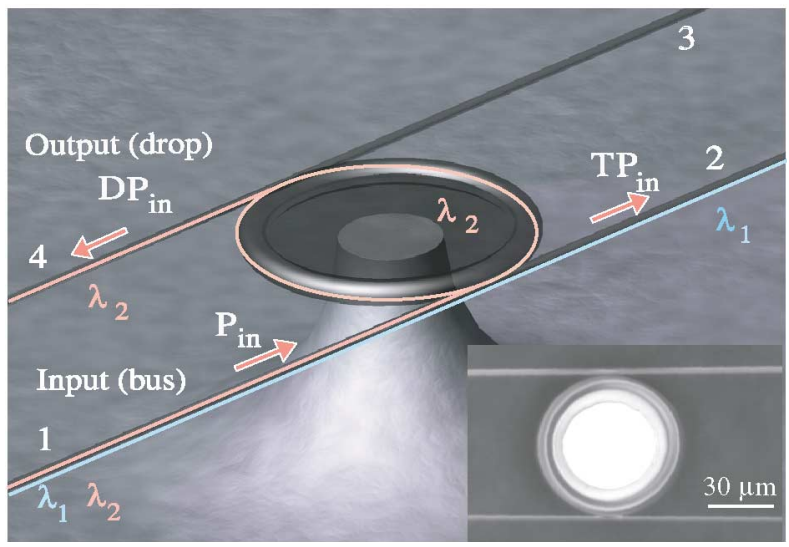

FIG. 1 (color). Illustration showing a microtoroid four port filter. Wavelength $\lambda_{2}$ in the bus waveguide is resonant with a whispering gallery mode of the resonator and subsequently couples to the drop waveguide. Nonresonant launched power $\left(\lambda_{1}\right)$ is transmitted past the resonator-fiber taper junction. The inset is a top view photomicrograph of a microtoroid coupled to two tapered optical fibers. 
assumption of weak coupling between the resonator and waveguides, which is valid in the current work. Weak coupling allows the separation of individual contributions to the cavity field decay time. A quality factor can be assigned to each dissipation process where the total quality factor takes the following compact form:

$$
\frac{1}{Q_{\text {total }}}=\frac{1}{Q_{0}}+\frac{1}{Q_{\text {bus }}}+\frac{1}{Q_{\text {drop }}} .
$$

$Q_{\text {bus }}$ and $Q_{\text {drop }}$ are quality factors associated with coupling to bus (input) and drop (output) waveguides, respectively. The overall quality factor of the system $\left(Q_{\text {total }}\right)$ determines the filter bandwidth and is lower than the intrinsic $Q$ of the cavity $\left(Q_{0}\right)$ due to the intentionally introduced couplings at the waveguide-resonator junctions. The following relationship exists between the transmitted and dropped power:

$$
\left[1+\frac{Q_{\text {drop }}}{Q_{0}}\right] D=1-T,
$$

where $T$ and $D$ are the transmitted and coupled (dropped) powers normalized to the incident signal power (see Fig. 1). Assuming a fixed coupling between the resonator and the drop waveguide (fixed $Q_{\mathrm{drop}}$ ), the dropped power to port 4 and the transmitted power to port 2 lie on a straight line, as coupling to the bus waveguide $\left(Q_{\text {bus }}\right)$ varies. At the critical coupling point $(T=0)$ [16,17], the drop efficiency reaches its maximum value:

$$
D_{\max }=1-\frac{2 Q_{\text {total }}}{Q_{0}}
$$

The important role of high intrinsic $Q$ cavities (high $Q_{0}$ ) in obtaining high power transfer efficiency is apparent in this expression. The condition of criticality $Q_{\text {bus }}^{-1}=$ $Q_{\text {drop }}^{-1}+Q_{0}^{-1}$ assures that coupling to the resonator from the input waveguide compensates both for the intrinsic loss of the cavity and the power coupling to the drop waveguide. It also ensures high transmission extinction of the resonant signal in the input waveguide. The sources of deviation from unity efficiency include the degree to which the resonator is noncritically coupled $(T>0)$, the high, but nonetheless finite intrinsic $Q$ factor of the resonator, and the parasitic losses at the resonatorwaveguide junction, which include both scattering losses and coupling to the undesired modes of the tapered fiber [14]. As is discussed later, the latter losses which can be effectively lumped into the $Q_{0}$ of the coupled resonator are negligible in this work. Also, throughput extinctions above $30 \mathrm{~dB}(T<0.001)$ are achieved which rules out the noncritical coupling as a significant source of power transfer inefficiency. Therefore, in this work the efficiency is solely limited by the finite $Q$ of the cavities.

To confirm the theoretical analysis, microtoroids of various sizes and quality factors were fabricated according to the previously reported microtoroid fabrication process [13]. Low-loss $(<0.3 \mathrm{~dB})$ fiber tapers, serving as input and drop waveguides, were simultaneously made by the technique of continuous flame heating and stretching two standard single-mode fibers. The device presented in this work is a microtoroid of principal diameter $65 \mu \mathrm{m}$ and minor diameter of approximately $6 \mu \mathrm{m}$. Fiber tapers used in the measurement had waist diameters of approximately 1 and $1.7 \mu \mathrm{m}$, for bus and drop waveguides, respectively. The chip containing the microtoroid was held on a platform that could be manipulated relative to the waveguides using a $10 \mathrm{~nm}$ stepresolution piezoelectric stage. To fulfill the assumption of constant coupling to the drop channel (fixed $Q_{\text {drop }}$ ), the fiber taper used for the drop waveguide was held in contact with the resonator. The other waveguide was manipulated using a probe controlled by a high-resolution micropositioner. A single-mode, tunable, external cavity laser, emitting in the $1550 \mathrm{~nm}$ band, was used to excite the whispering-gallery modes of the microtoroid. Transmission and drop powers were simultaneously monitored using two $(125 \mathrm{MHz})$ photodiode detectors, as the laser frequency was slowly $(<10 \mathrm{~Hz})$ scanned over about $50 \mathrm{GHz}$ using a function generator. Before discussing the experimental results it should be noted that in the present work the power transfer efficiency has reached such a high level that uncertainties in power measurements associated with losses external to the device such as splice losses are vastly larger than the resonator-based transfer loss itself. In this regard, external losses incurred by mode conversion from a single-mode fiber to the thin waveguides at the resonator coupling region can be excluded in the device efficiency measurements. This is done by using ports 1 and 2 as input ports for the device in separate measurements and subtracting off the individual losses of the bus and drop fiber taper mode converters (about $0.15 \mathrm{~dB}$ per converter). Using this technique accuracy in transfer loss measurements of about $0.1 \mathrm{~dB}(\sim 3 \%)$ can be achieved. Since precision of efficiency measurements is limited to this level, in order to verify our theoretical predictions we opt to measure the efficiency in more weakly loaded toroid filters having correspondingly higher $Q_{\text {total }}$ factors and hence lower transfer efficiencies [see Eq. (3)]. To this end, waveguides with larger waist diameters can be used to limit the extent of the evanescent field and thereby reduce the waveguideresonator coupling.

Coupling to the resonator by each fiber taper acting alone was first examined. Resonator linewidth measurements using a single waveguide in the highly undercoupled regime $\left(Q_{\text {total }} \cong Q_{0}\right)$ revealed a $3 \mathrm{~dB}$ bandwidth of about $1.9 \mathrm{MHz}$ at the resonant wavelength of $1565 \mathrm{~nm}$, which translates to an intrinsic resonator quality factor of $1 \times 10^{8}$. Overcoupled $\left(Q_{\text {bus }}<Q_{0}\right)$ transmissions in excess of $96 \%$ were observed, which according to Ref. [14] place a lower bound on junction ideality of better than $99 \%$ in the overcoupled regime (less than $1 \%$ of power is coupled to undesired modes of the tapered fiber). 


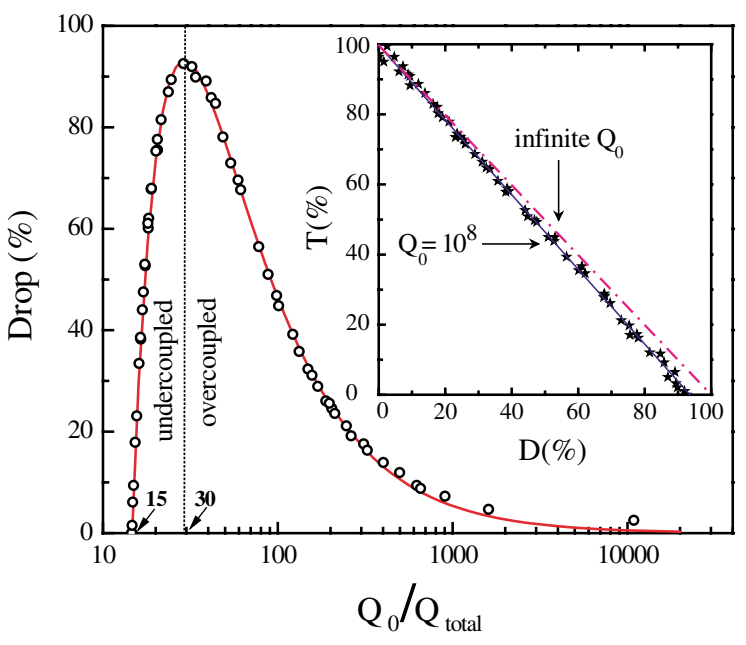

FIG. 2 (color). Transferred power to the drop waveguide $(D)$ versus the inverse-loaded $Q$ of the system. The data show a factor of 15 reduction in the $Q$ factor upon attachment of the drop taper to the resonator. At the critical coupling point $\left(Q_{0} / Q_{\text {total }}=30\right), 93 \%$ of the input power is transferred to the drop port and throughput transmission vanishes. The theoretical drop efficiency is given by the solid line. The inset is a plot of $T$ vs $D$ using the same data in the main figure which verifies their linear relationship. The solid curve is the theoretical curve and the dashed curve gives the ideal case of infinite intrinsic $Q$.

Figure 2 shows the drop efficiency $D$ (the fraction of power at the first waveguide redirected to the second waveguide) measured for different loading regimes and plotted versus the ratio of the intrinsic $Q$ to the loaded quality factor of the system. Data were measured as the gap between input waveguide and resonator was varied. As noted earlier, the drop waveguide coupling is fixed during these measurements. As an aside, note that the total quality factor is reduced by a factor of 15 (labeled in Fig. 2) upon attachment of the drop waveguide to the resonator. The $Q$ factor at the critical coupling point is a factor of 30 lower than the intrinsic $Q\left(=10^{8}\right)$, corresponding to a filter bandwidth of $57 \mathrm{MHz}$. From Eq. (3), the factor of 30 yields a maximum theoretical drop efficiency of $93.3 \%$ ( $0.3 \mathrm{~dB}$ loss) in good agreement with the measured values in Fig. 2. The inset in Fig. 2 is a plot of the measured transmission versus the drop power and confirms the predicted linear relationship. This way of plotting the data results in a more reliable estimate of the maximum drop efficiency, which is $(94 \pm 3) \%$ for this device. This is to our knowledge the best efficiency ever reported for a four port microcavity-type resonant coupler. It also agrees well with the expected theoretical value of $93.3 \%$. The transmission data are obtained by normalizing the on-resonance transmission to its value with the resonator far from the waveguides. The nonresonant insertion loss in the bus channel was measured to be less than $0.02 \%$ during the experiment. This loss, as noted earlier, is due to scattering of the evanescent field at

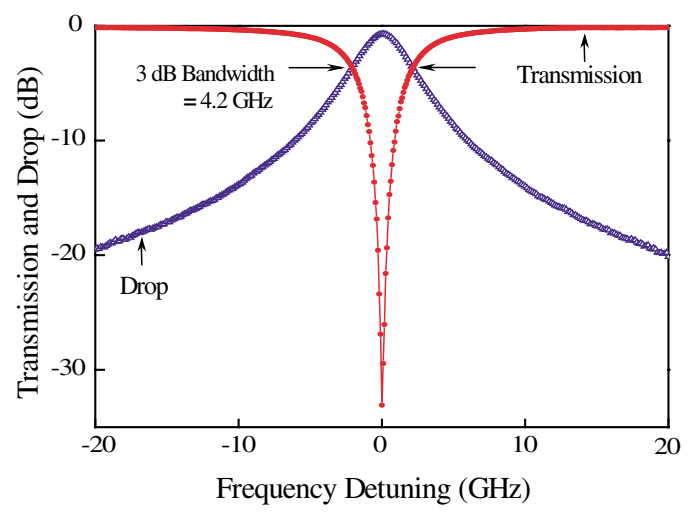

FIG. 3 (color). Drop port (triangles) and throughput port (circles) spectra measured for a four port resonant coupler with $4.2 \mathrm{GHz}$ bandwidth. Extinction of $33 \mathrm{~dB}$ in input channel occurs at the resonant wavelength of $1540 \mathrm{~nm}$.

the waveguide-resonator junction and is measured by comparing the off-resonance transmission in port 2 , when the device is working as an add-drop, to the same transmission but when the resonator is far from the bus waveguide (infinite gap). As such, the measurement of insertion loss yields a higher level of accuracy and precision than is possible for measurement of the drop efficiency.

The small deviation from the ideal case of infinite intrinsic $Q$ can be observed in the inset of Fig. 2 (see the dashed line). This deviation, a direct consequence of energy conservation, is due to the power dissipation in the cavity due to finite intrinsic losses. Note that in the theoretical model all parasitic losses have been lumped into $Q_{0}$. Therefore the excellent agreement between the measured values for the efficiency and the theoretical predictions, which assume all the parasitic losses come from the intrinsic loss of the cavity, is further evidence of low loss at the waveguide-resonator junctions. Figure 2 also shows that the bus waveguide can induce at least a factor of $10^{3}$ decrease in the quality factor relative to that of the original resonator $\left(Q_{0}=10^{3} Q_{\text {bus }}\right)$. Were the drop waveguide designed to produce this level of coupling, an exceedingly high drop efficiency of $99.9 \%$ would result with an overall loaded $Q$ factor of $5 \times 10^{4}$ or a filter bandwidth of about $4 \mathrm{GHz}$. Higher bandwidths in this range are of interest in telecommunication applications of these devices [18-20].

Figure 3 shows transmission and drop spectra measured for a device designed to operate in the higher bandwidth regime. The filter shape is Lorentzian and the bus waveguide extinction is a record $33 \mathrm{~dB}$. Note that the FWHM of the drop and transmission spectra almost coincide due to a negligible amount of loss in power transfer.

The results presented in this work demonstrate the ability of taper-coupled ultrahigh- $Q$ toroid microcavities to reach exceedingly high power transfer efficiencies. 
This can be of great interest in the study of processes requiring very low loss. As an example, losses are one of the main impediments in realizing long-distance quantum communication networks, as they tend to destroy the entanglement of quantum states and ultimately decrease the communication fidelity [21]. Also the ability to filter out broadband noise while maintaining the signal amplitude (low insertion loss) is highly desirable in radiofrequency photonics [22] or sensitive power detection applications (e.g., single photon experiments). The latter application requires high finesse cavities to achieve strong, out-of-band noise rejection. A free spectral range of about $8 \mathrm{~nm}$ and loaded $Q$ of $3.3 \times 10^{6} \mathrm{imply}$ a finesse in excess of $10^{4}$ for the device studied here. On the other hand, due to the extremely low intrinsic loss of cavities in these devices, the quality factor of the system is almost entirely determined by coupling to waveguides. Therefore a remarkable operational dynamical range (both bandwidth and power transfer ratio) is attainable by controlling the coupling between resonator and individual waveguides. Finally, the excellent agreement of the experimental results with theoretical predictions suggests that this method allows the realization of resonant couplers with negligible losses. Indeed, the selection of filter design in the present study was chosen so as to produce a measurable loss within the accuracy of our experimental equipment for purposes of comparison to the model.

This work was supported by DARPA, by the Caltech Lee Center, and by the National Science Foundation. The author acknowledges T. J. Kippenberg, D. K. Armani, and S. M. Spillane for helpful discussions.

[1] H. J. Kimble, Phys. Scr. T76, 127 (1998).

[2] S. Haroche, Phys. Scr. T76, 159 (1998).
[3] A. Kiraz et al., Appl. Phys. Lett. 78, 3932 (2001).

[4] J. I. Cirac, P. Zoller, H. J. Kimble, and H. Mabuchi, Phys. Rev. Lett. 78, 3221 (1997).

[5] J. M. Gerard et al., Physica (Amsterdam) 2E, 804 (1998).

[6] E. Waks et al., Nature (London) 420, 762 (2002).

[7] D.W. Vernooy, A. Furusawa, N. P. Georgiades, V.S. Ilchenko, and H. J. Kimble, Phys. Rev. A 57, R2293 (1998).

[8] V. B. Braginsky, M. L. Gorodetsky, and V.S. Ilchenko, Phys. Lett. A 137, 393 (1989).

[9] B. E. Little, S. T. Chu, W. Pan, D. Ripin, T. Kaneko, Y. Kokubun, and E. Ippen, IEEE Photonics Technol. Lett. 11, 215 (1999).

[10] S. Suzuki, Y. Hatakeyama, Y. Kokubun, and S. T. Chu, J. Lightwave Technol. 20, 745 (2002).

[11] K. Djordjev, S. J. Choi, and P. D. Dapkus, IEEE Photonics Technol. Lett. 14, 1115 (2002).

[12] P. Rabiei, W. H. Steier, C. Zhang, and L. R. Dalton, J. Lightwave Technol. 20, 1968 (2002).

[13] D. K. Armani, T. J. Kippenberg, S. M. Spillane, and K. J. Vahala, Nature (London) 421, 925 (2003).

[14] S. M. Spillane, T. J. Kippenberg, O. J. Painter, and K. J. Vahala, Phys. Rev. Lett. 91, 043902 (2003).

[15] A. Vorckel, M. Monster, W. Henschel, P. H. Bolivar, and H. Kurz, IEEE Photonics Technol. Lett. 15, 921 (2003).

[16] M. Cai, O. J. Painter, and K. J. Vahala, Phys. Rev. Lett. 85, 74 (2000).

[17] A. Yariv, IEEE Photonics Technol. Lett. 14, 483 (2002).

[18] B. J. Offrein et al., IEEE J. Sel. Top. Quantum Electron. 5, 1400 (1999).

[19] R. Grover et al., Opt. Lett. 26, 506 (2001).

[20] B. E. Little, S. T. Chu, H. A. Haus, J. Foresi, and J. P. Laine, J. Lightwave Technol. 15, 998 (1997).

[21] S. J. van Enk, J. I. Cirac, and P. Zoller, Phys. Rev. Lett. 78, 4293 (1997).

[22] X. S. Yao and L. Maleki, J. Opt. Soc. Am. B 13, 1725 (1996). 